\title{
1 Diverse and rapidly evolving viral strategies modulate arthropod vector gene expression
}

3

$6{ }^{1}$ Okinawa Institute of Science and Technology, 1919-1 Tancha Onna-son, 904-0495

7 Okinawa, Japan

$8{ }^{2}$ Australian National University, Canberra, ACT, 2600, Australia

10 Corresponding Authors *

11 Nurit Eliash norikachan@gmail.com;

12 Alexander S. Mikheyev alexander.mikheyev@anu.edu.au 


\section{Abstract}

15 Vector-borne viral diseases threaten human and wildlife worldwide. Vectors are often

16 viewed as a passive syringe injecting the virus, however to survive, replicate and spread,

17 viruses must manipulate vector biology. While most vector-borne viral research focuses on

18 vectors transmitting a single virus, in reality vectors often carry diverse viruses. Yet how

19 viruses affect the vectors remains poorly understood. Here we focused on the varroa mite,

20 an emergent parasite that vectors over 20 honey bee viruses, and has been responsible for

21 colony collapses worldwide, as well as changes in global viral populations. Co-evolution of

22 the varroa and the viral community makes it possible to investigate whether viruses affect

23 vector gene expression, and whether these interactions affect viral epidemiology. Using a

24 large set of available varroa transcriptomes we identified how abundances of individual

25 viruses affect the vector's transcriptional network. Perhaps surprisingly, we found no

26 evidence of competition between viruses, but rather that some virus abundances are

27 positively correlated. Furthermore, we found a strong correlation between the extent to

28 which a virus interacts with the vector's gene expression and co-occurrence with other

29 viruses, suggesting that interactions with the vector affect epidemiology. We

30 experimentally validated this observation by silencing candidate genes using RNAi and

31 found that reduction in varroa gene expression was accompanied by a change in viral load.

32 Combined, the meta-transcriptomic analysis and experimental results shed light on the

33 mechanism by which viruses interact with each other and with their vector to shape the

34 disease course.

36 Key words: vector-virus interaction, gene network analysis, varroa, RNAi silencing 


\section{Introduction}

38 Vector borne viral diseases are infections caused by viruses and transmitted by infected 39 arthropods (vectors) such as mosquitoes, ticks and mites (Brault et al., 2018; de la Fuente et al., 2017). They drive species evolution in both natural and man-managed ecosystems, and impose a great threat to organisms across kingdoms. Most disease-causing plant viruses depend completely on vectors for spread and survival (Ng \& Falk, 2006; Whitfield et al., 2015), but animals also suffer from vector borne viruses (Olival et al., 2017). For humans, these include Dengue, Chikungunya, Chagas disease, Japanese encephalitis, Zika, and yellow fever, leading to hundreds of thousands of deaths worldwide especially in developing countries (Weaver et al., 2018; World Health Organization, 2017). Moreover, ongoing globalization processes and climate change are expected to increase their outbreak frequency (Rocklöv \& Dubrow, 2020; Sutherst, 2004). The most efficient and sustainable measure of coping with emerging vector-borne diseases is to control their vectors (A. L. Wilson et al., 2020).

51 Vectors are often viewed as merely a passive syringe injecting the virus. However, to

52 promote replication and transmission viruses must regulate the vector's immune system and behaviour, which requires interaction with the vector. Indeed, former studies showed that viral infection can alter the vector feeding behaviour, fecundity, longevity and survival (Jackson et al., 2012; Maciel-de-Freitas et al., 2011; Moncayo et al., 2000; Neelakanta et al., 2010). The molecular mechanism underlying these interactions was studied in both cell cultures (Cime-Castillo et al., 2015; Göertz et al., 2019; Schnettler et al., 2014), and in vivo experiments (Luplertlop et al., 2011; Zink et al., 2015), revealing vector immune-related genes whose expression is regulated following viral infection, and show that a crosstalk between vector genes and the virus is imminent for successful viral replication and transmission (Huang et al., 2019).

62 Most of the studies have investigated the interaction of a vector with a single virus (Huang 63 et al., 2019), and a few considered up to two co-occurring viruses (Goenaga et al., 2015;

64 Göertz et al., 2017). However, vectors can usually carry diverse viruses that may interact 65 with each other (Batovska et al., 2019; Batson et al., 2021; Ciota, 2019; Vogels et al., 2019). 
67 such as virulence and transmission, either by direct virus-virus interaction, or indirectly by competition or cooperation (Erez et al., 2017; Ferguson et al., 2003; Nickbakhsh et al., 2019). Therefore, multi-infection may have a profound effect on viral evolution, diversity and pathogenicity (Alcaide et al., 2020; Díaz-Muñoz, 2017). In contrast to these studies, the effect of multiple infections on vectors has received far less attention, although is expected to have considerable effect on the disease epidemiology

73 (Ciota, 2019; Vogels et al., 2019). Therefore, virus-virus interaction is one of the main

74 challenges in virology today (Sanjuán et al., 2021). We can expect that similar to the case of a host co-infected by several viruses, multi-infection will also have an effect on the vectorvirus interaction. Yet we cannot simply infer from host-virus studies to vector-viruses, as the two are undergoing different evolutionary processes: while host-pathogen interactions are antagonistic by definition, vector-virus interaction are less definite and can fall anywhere on the continuum between antagonistic and mutualistic (A. J. Wilson et al., 2017). Therefore, we don't know how vector-borne viruses interact, and how this may affect their relationship with their vector.

Varroa destructor (hereafter "varroa") is a parasitic mite which vectors honeybee pathogenic viruses, and is routinely co-infected by multiple viruses (McMenamin \& Genersch, 2015). The introduction of varroa to the European honey bee (Apis mellifera) has dramatically changed the honeybee viral landscape, leading to worldwide colony collapses threatening global food security (Steinhauer et al., 2018). In varroa-free colonies the viral diversity is high, with somewhat low levels (Carreck et al., 2010). Normally, one-two years following varroa introduction to a naive colony, viruses diversity and titers are shifting greatly, giving rise to high levels of a few viral strains as the colony is dwindling to its final collapse (Martin et al., 2012). Despite these observations, not much attention was given to varroa-virus interaction, and how it may explain the varroa key-role as a vector of

93 honeybee viral disease. In addition, varroa carries over 20 diverse viruses commonly co94 occurring (Table 1), including bee-pathogenic viruses, of which some are highly associated 95 with varroa, while others are of unknown pathogenicity to either varroa or bee (Yañez et al., 2020). Therefore, the varroa-virus system is a unique opportunity to investigate how different viruses interact with each other and with their vector. 
98 Here we studied the interaction of diverse viruses with varroa's transcriptional network,

99 hypothesizing that different viruses have distinct effects. We explored a large set of RNAseq

100 data from the vector aiming (1) to determine if viruses compete or cooperate in their

101 vector, and (2) to test whether interactions between individual viruses and the vector

102 correlate with viral epidemiological traits, specifically co occurrence with other viruses. We

103 found no evidence of competition between viruses, but positive correlations in the

104 abundance of specific viruses. Furthermore, we found a strong correlation between the

105 extent to which a virus interacts with the vector's gene expression and its overall

106 prevalence, and have experimentally validated some of these predictions. These results

107 show that multi-viral infection can occur not only in the host, but also in the vector, and

108 that these interactions have implications on the virus relationship with its vector and

109 therefore on the disease course. 
bioRxiv preprint doi: https://doi.org/10.1101/2021.12.16.472853; this version posted December 17, 2021. The copyright holder for this preprint (which was not certified by peer review) is the author/funder, who has granted bioRxiv a license to display the preprint in perpetuity. It is made available under aCC-BY-NC-ND 4.0 International license.

Table 1. Most of the known bee viruses are present in both honey bee and mite, but only a few replicate in the mite, or shown to be varroa-vectored. Virus presence in bees and varroa, and its ability to replicate and vectored by the mite between bees are marked by: $(+)$ confirmed; $(--)$ not demonstrated; (S) suspected; (?) unknown. A star mark $\left(^{*}\right)$ next to the virus abbreviated name indicates that this virus was not detected in any of the varroa libraries in the current study. More details on each virus sequence and references can be found in Table S1.

\begin{tabular}{|c|c|c|c|c|c|c|c|c|}
\hline Type & Family & $\begin{array}{l}\text { Abbreviated } \\
\text { name }\end{array}$ & Full name & $\begin{array}{l}\text { Present } \\
\text { in bee }\end{array}$ & $\begin{array}{l}\text { Replicate } \\
\text { in bee }\end{array}$ & $\begin{array}{l}\text { Present } \\
\text { in varroa }\end{array}$ & $\begin{array}{l}\text { Replicate } \\
\text { in varroa }\end{array}$ & $\begin{array}{l}\text { Vectored } \\
\text { by varroa }\end{array}$ \\
\hline \multirow{17}{*}{ ssRNA(+) } & \multirow{6}{*}{ Iflaviridae } & DWVa & Deformed wing virus type a & + & + & + & -- & + \\
\hline & & DWVb & Deformed wing virus type $b$ & + & + & + & + & + \\
\hline & & DWVc & Deformed wing virus type $c$ & + & $?$ & + & ? & $?$ \\
\hline & & SBPV & Slow bee paralysis virus & + & + & + & $?$ & + \\
\hline & & SBV & Sacbrood virus & + & + & + & s & s \\
\hline & & VDV2 & Varroa destructor virus 2 & -- & -- & + & ? & -- \\
\hline & \multirow{4}{*}{ Dicistroviridae } & IAPV & Israel acute paralysis virus of bees & + & + & + & + & + \\
\hline & & KBV & Kashmir bee virus & + & + & + & + & + \\
\hline & & ABPV & Acute bee paralysis virus & + & + & + & -- & s \\
\hline & & BQCV & Black queen cell virus & + & + & + & + & $?$ \\
\hline & \multirow{2}{*}{ Tymoviridae } & BMV & Bee Macula-like virus & + & + & + & ? & s \\
\hline & & VTLV & Varroa Tymo-like virus & + & $?$ & + & ? & $?$ \\
\hline & Flaviviridae & $\mathrm{AFV} *$ & Apis flavivirus & + & $?$ & s & ? & $?$ \\
\hline & \multirow{4}{*}{$\begin{array}{l}\text { Unclassified } \\
\text { ssRNA(+) }\end{array}$} & LSV & Lake Sinai virus & + & + & + & ? & s \\
\hline & & VDV3 & Varroa destructor virus 3 & -- & -- & + & $?$ & -- \\
\hline & & CBPV * & Chronic bee paralysis virus & + & + & + & + & -- \\
\hline & & ANV * & Apis mellifera nora virus 1 & + & ? & ? & $?$ & $?$ \\
\hline \multirow{3}{*}{$\operatorname{ssRNA}(-)$} & Rhabdoviridae & ARV-2 & Apis mellifera rhabdovirus-2 & + & + & + & s & s \\
\hline & Orthomyxoviridae & VOV-1 & Varroa orthomyxovirus-1 & + & + & + & + & $?$ \\
\hline & $\begin{array}{l}\text { Unclassified } \\
\text { ssRNA(-) }\end{array}$ & VDV4 & Varroa destructor virus 4 & + & ? & + & ? & $?$ \\
\hline \multirow{3}{*}{ DNA } & Genomoviridae & VPVL_36* & $\begin{array}{l}\text { Varroa mite associated genomovirus } 1 \\
\text { isolate VPVL_36 }\end{array}$ & $?$ & ? & + & ? & $?$ \\
\hline & $\begin{array}{l}\text { Unclassified } \\
\text { dsDNA }\end{array}$ & AmFV & Apis mellifera Filamentous virus & + & ? & + & $?$ & $?$ \\
\hline & $\begin{array}{l}\text { Unclassified } \\
\text { ssDNA }\end{array}$ & VPVL_46* & $\begin{array}{c}\text { Varroa mite associated virus } 1 \text { isolate } \\
\text { VPVL_ } 46\end{array}$ & $?$ & $?$ & + & $?$ & $?$ \\
\hline
\end{tabular}




\section{Results:}

\section{The viral landscape is heterogeneous across varroa libraries}

122 We found that each of the final 66 varroa RNAseq libraries contain at least three types of

123 viruses (Fig 1 and Table 1 ). The most prevalent viruses belong to the Iflaviridae family,

124 including the bee pathogenic viruses DWVa and DWVb, and the varroa-specific virus, VDV2.

125 On the other hand, five viruses whose presence in varroa was suspected or reported

126 before, were not detected in any of the libraries (CBPV, AFV, ANV, VPVL_46 and VPVL_36)

127 (Fig 1 and Table 1). Viral load homogeneity across libraries varied greatly between viruses:

128 a few viruses were extremely variable (e.g, DWVa was not detected in a few libraries while

129 reaching < 400,000 TPMs in others), while other viruses (such as ARV-2, VOV-1 and VDV2),

130 were found in somewhat similar loads in most libraries. Interestingly, VDV2 is the only

131 virus that was present in all varroa libraries. 


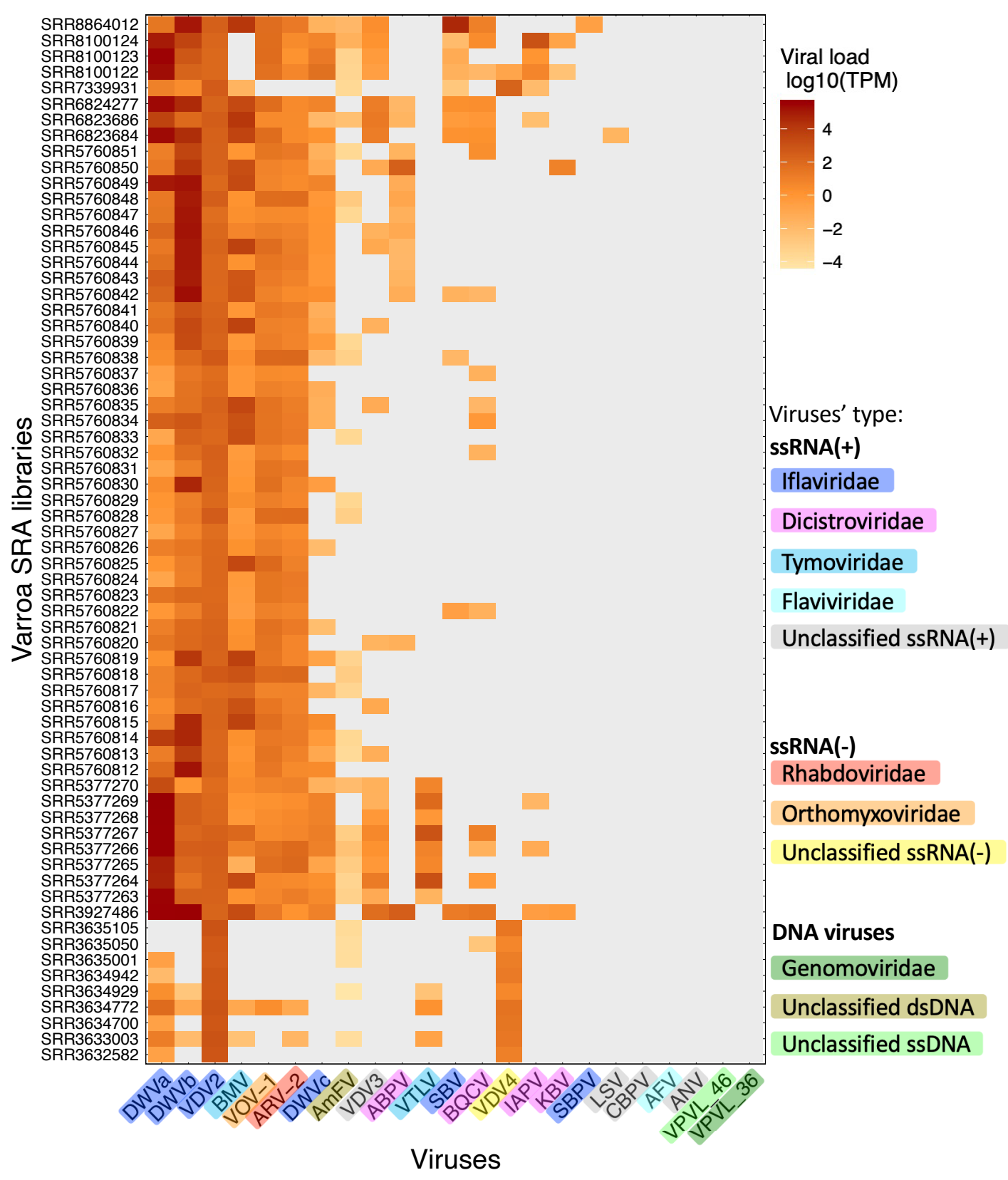

Figure 1. Viral load is diverse across the different varroa libraries. Members of the Iflavirus family are the most prevalent, yet while some viruses are homogenous across libraries (VDV2), others are highly diverse (e.g. DWVa and DWVb). Values are log10 transformed of the reads' TPM (transcript per millions). Zero values are marked in grey (i.e., none of the reads in this library mapped to this virus). The viruses' names are abbreviated as described in Table 1. 
No evidence for competition between the different viruses

Multi-infection by several viruses raises the obvious option of interactions between them.

(Pearson correlation followed by Benjamini-Hochberg FDR-correction, $\mathrm{P}<0.1$ ) (Fig 2a).

144

\section{a. Virus-virus interaction}

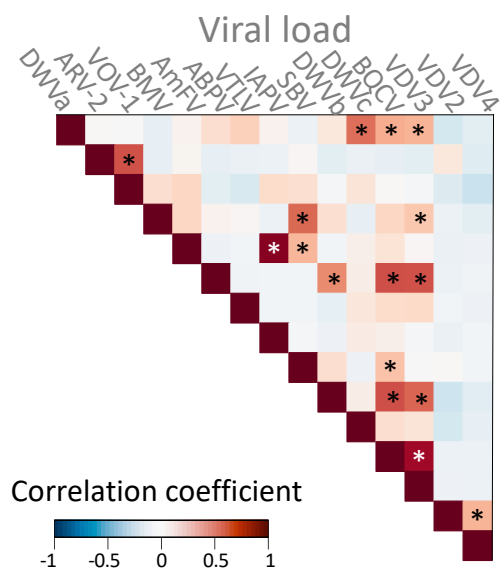

\section{b. Varroa-virus interaction}

Varroa modules 115423135106912811147

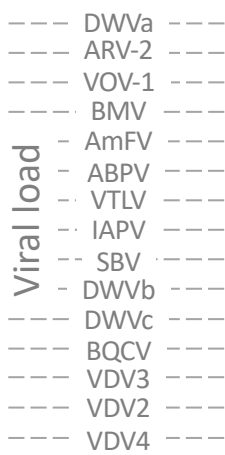
**

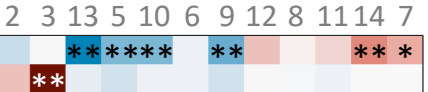

c. Correlation between (a) virus-virus interaction and (b) varroa-virus interaction

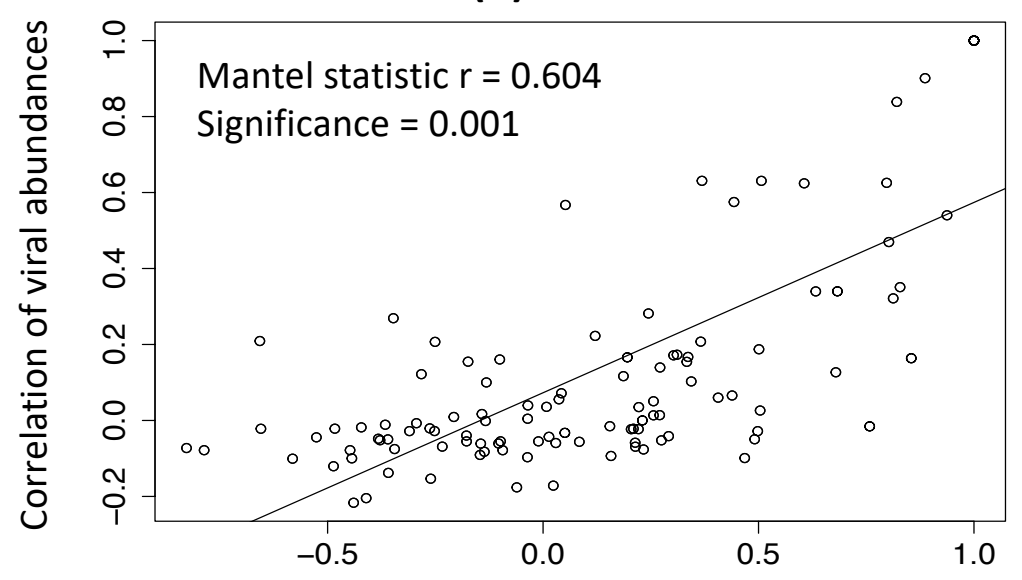

Correlation of viral interaction with varroa modules

Figure 2. Intra-viral interactions can predict virus - vector interactions. a. Correlation between viruses' loads. b. Correlation between viruses' loads and varroa modules (eigengenes). c. Correlation model between viral load correlations (a), and the distance-matrix of the module-virus correlations (b). In figures a and $b$, viruses and modules are ordered according to hierarchical clustering; $P$ values of the Pearson coefficient in figures $\mathrm{a}$ and $\mathrm{b}$ are adjusted according to FDR-correction; correlation significance marked by $\left(^{*}\right) 0.1<\mathrm{P}<$ $0.01 ;(* *) \mathrm{P}<0.01$. For analysis in figure $\mathrm{c}$, Mantel test for correlation between two matrices was conducted using 1,000 permutations. All viruses' loads are transformed by $\log 10$ of the (TPM), Transcripts Per Million. 


\section{Varroa modules interact with its viral load}

184 Gene network analysis of the 66 varroa RNAseq libraries clustered the 10,247 varroa genes into 15 modules, each module containing co-expressed genes. The modules were numbered from the largest (module number one which contains 3,625 co-expressed genes) to the smallest (module number 15 which contains 31 genes) (Fig S1a and b). Correlating varroa modules eigengenes to their viruses' loads (Transcript Per Million, TPM), we found significant interactions between specific modules and viruses (Pearson correlation followed by Benjamini-Hochberg FDR-correction, $\mathrm{P}<0.1$, Fig 2b). The interaction direction and strength, represented by the correlation coefficient, varied between virus-module pairs. While VDV2, VDV4, ARV-2 and VOV-1 show positive interaction with some modules (modules 3, 6 and 9), 'bee-viruses' (such as DWVc and BQCV) show negative interaction with a different module (module 13). Interestingly, the only virus that shows both negative and positive interactions with vector's modules is the bee-pathogenic virus, DWVa.

\section{GO-terms of interacting modules}

The significantly interacting modules consist of genes that are enriched for regulatory related GO-terms such as "Regulation of gene expression" (module 10), "Regulation of metabolic processes" (modules 6 and 9) and immune response related GO-terms, such as "Immunoglobulin production" G0:0002377 (module 6). In addition, modules 3 and 6 are

202 enriched for viral and symbiont related GO-terms, such as "viral process" G0:0016032 and "modulation by virus of host process" G0:0019054, and "Regulation of biological process involved in symbiotic interaction", G0:0043903. For the list of all significant GO-terms in the significantly interacting modules please see Tables S2-S10 (available on https://nuriteliash.github.io/varroa-virus-networks/). Of the 17 RNAi-gene homologs recently

207 identified in varroa (Beatrice T. Nganso et al., 2020), we found that 13 genes belong to module 3 (positively interacting with ARV-2 and VOV-1) (Table S11). 


\section{Viruses that are found together interact with the vector's gene coexpression modules}

\section{2 in similar ways}

213 Among the module-virus interactions (Fig 2b), we can detect viruses that share a similar

214 interaction with the same module. For example, VDV2 and VDV4 abundance positively

215 correlated with modules 6 and 9. Interestingly, abundances of these two viruses also

216 significantly correlate with each other (Fig 2a). Similarly, ARV-2 and VOV-1 also have

217 positively correlated abundances and positive interactions with module 3 (Fig 2a and 2b).

218 Likewise, DWVa, DWVc, BQCV and VDV3 are positively correlated in abundance, and all

219 four are negatively correlated to module 13 . We evaluated if this pattern between virus-

220 virus interaction and virus-module interaction can be generalized across all virus-virus-

221 module interactions in our data set. Indeed, we found a significant positive correlation

222 between the two distance matrices (Mantel-test for correlation between two distance-

223 matrices (Mantel, 1967), Mantel statistic $r=0.604, p=0.001$ ) (Fig 2c). In other words,

224 there is a correlation between viral co-occurrence and the manner in which they interact

225 with the vector.

Validating varroa-virus interaction: gene silencing is accompanied by a change in viral load

To experimentally validate the varroa-virus interaction as predicted by the gene-network analysis, we silenced the mite's genes using RNAi and tested its viral load, compared to the control (GFP-treated mites). For silencing, we selected candidate genes based on both the gene-network analysis and on the literature. From module 10, which interacts with the

234 the module. In addition, these genes also possessed a relevant annotation, based on

235 literature survey and / or the presence of conserved domain in the predicted coded protein

236 (e.g. immune response related domains, and genes that were previously reported to

237 interact with host/vector) (for the list of silenced genes, their module-membership and

238 annotation, see table S12). Four of the five tested genes were successfully silenced, i.e., for

239 these genes a significant reduction in relative gene expression was measured in mites

240 treated with dsRNA, compared to control mites $(\mathrm{P}<0.05$, Wilcoxon signed-ranks test,

241 followed by FDR-correction, table S13). We then compared the relative viral loads of DWVa, 
242 VDV2 and ARV-2 using quantitative PCR (qPCR). DWVa was predicted to negatively

243 interact with the genes in module 10, while VDV2 and ARV-2 were not predicted to interact

244 with the module (Fig 2b). Of all silenced genes, only mites that were treated with dsRNA of

245 Cuticle protein type 8-like gene (short name: CuP8, accession: LOC111248360) showed a

246 significant change in viral load, compared to control mites. Mites with decreased

247 expression of CuP8 had lower VDV2 and ARV-2 viral loads, compared to control mites (P =

248 0.02, Wilcoxon signed-ranks test, followed by FDR-correction), while DWVa viral load has

249 also decreased, but not significantly ( $\mathrm{P}=0.2$, Wilcoxon signed-ranks test, followed by FDR-

250 correction) (Fig 3a and 3b).

251 The dsRNA soaking treatment did not affect mite survival, compared to the control GFP-

252 dsRNA treated mites (Fisher exact test for goodness of fit, p < 0.05) (Table S14).

253

a. Gene expression

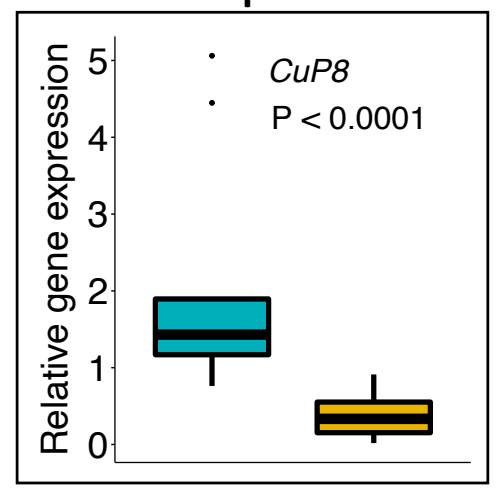

b. Viral load

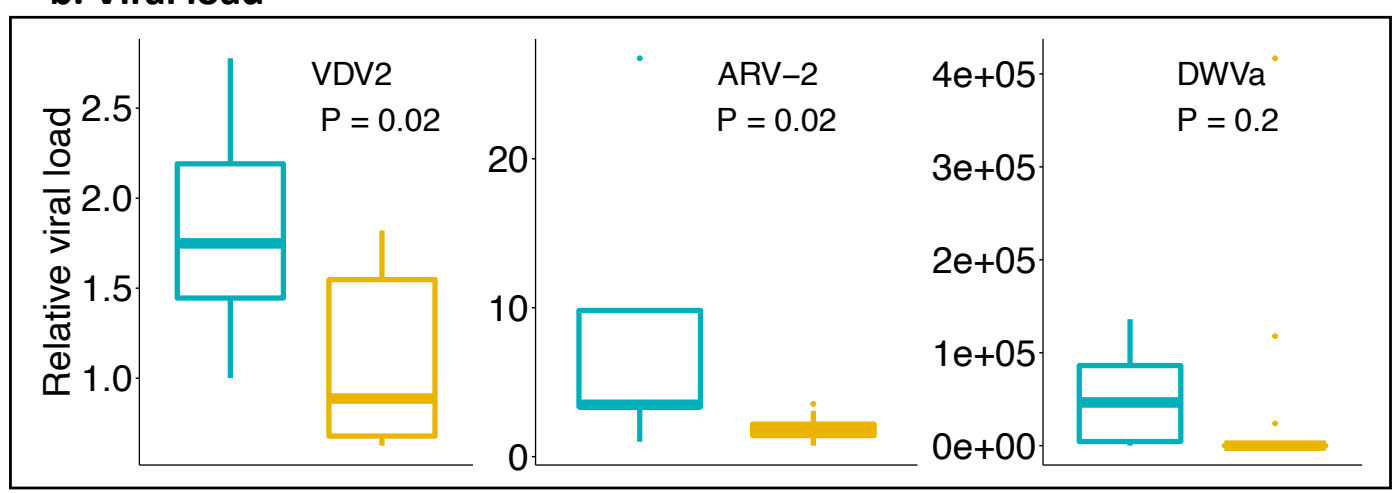

254 Figure 3. Validating varroa - virus interaction using gene-silencing (RNAi). a. Relative

255 expression of $C u P 8$ gene in control (GFP-dsRNA, n=9) and silenced mites (treated with $C u P 8$ -

256 dsRNA, $n=13$ ). b. Relative viral load of VDV2, ARV-2 and DWVa in control and silenced mites.

257 Significant difference between control and silenced mites was tested using Wilcoxon signed-ranks

258 test, followed by FDR-correction. The boxplot shows the interquartile of the data values, the inner

259 thicker line of the box represents the median value, and the dots are potential outliers. 


\section{Discussion}

261 Vector-borne viruses rely on another organism for transmission. As the vector plays a key

262 role in viral fitness, we hypothesized that different viruses have distinct effects on a vector,

263 observable as changes in gene expression. This was indeed true for viruses associated with

264 varroa mites. Namely, titers of individual viral species were associated with specific

265 changes to the vector's gene expression network (Fig 2b). Interestingly, co-occurring

266 viruses affected the vector gene expression network in similar ways (Fig 2c), suggesting a

267 link between viral epidemiological traits and its relationship with the vector. We further

268 propose that vector-virus interactions are evolving rapidly, as we found that closely related

269 viruses have distinct effects on the vector's gene expression. Interestingly, we found no

270 evidence of viral competition within the vector. On the contrary, abundances of some

271 viruses are positively correlated in co-infected vectors, suggesting a potential for viral

272 cooperation. These complex dynamics underscore the role of vector-virus interactions for

273 viral fitness.

Viruses interact with specific modules in the vector's transcriptional network

276 As we hypothesized, viral presence affects vector gene expression. We found that specific

277 modules in the vector's gene expression network respond to changes in viral titers in a

278 species-specific way (Fig 2b). This indicates that the host responds to viral presence. Some

279 of these responses may represent antiviral defenses (Göertz et al., 2019; Huang et al., 2019;

280 Schnettler et al., 2014; Zink et al., 2015) or general stress responses (Rosche et al., 2021)

281 that limit damage to the vector, though it is also possible that viruses trigger additional

282 responses in a way that benefits their spread.

283 In accordance with this work, we found that the interacting modules involve both specific

284 antiviral and non-specific stress responses. The modules included genes within the RNAi

285 pathway (see Table S11), the main arthropod antiviral response (Blair \& Olson, 2015).

286 These modules were enriched for infection-specific GO-terms such as immune response

287 and viral replication. At the same time, the modules were also enriched for regular cell

288 maintenance GO-terms such as cell metabolism, and gene expression regulation. It should

289 be noted that most of the modules responding to viral infection are not obviously related 
either to stress or antiviral gene expression, and their function vis a vis vector or viral fitness is unclear.

293 Following the network analysis, we have empirically validated the module-virus

294 interactions using gene-silencing experiments of genes with high module connectivity, 295 these are 'hub-genes' (Langfelder \& Horvath, 2008). While we found that experimentally 296 altering host gene expression does affect viral titre, the interactions were not in the 297 direction the network analysis predicted. Namely we found that the knockdown of CuP8, a 298 cuticular protein that has been found to assist in plant viral transmission by binding to 299 viruses (Deshoux et al., 2018), was accompanied by a significant reduction in viral load of VDV2 and ARV-2, and non-significant reduction in DWVa (Fig 3b). Yet, CuP8 was a hubgene in a module negatively correlated with DWVa (Fig 2b), suggesting that it's expression should be negatively correlated with that of the virus. The experimental result verifies on one hand the importance of the network hub-genes in the vector-virus interaction, while on the other hand, it illustrates the high complexity of the molecular mechanism underlying the vector response, which may involve other factors such as gene-to-gene transcriptional regulation, and interaction with the environment other microorganisms (Ciota, 2019).

\section{A link between viral epidemiological traits and their relationship with the vector} Interestingly, viruses with similar effect on the vector's transcriptional network had tended to co-occur (Fig 2c). This observation can be explained by two non-mutually exclusive explanations. On one hand, high titres of viral RNA and proteins can trigger defense or stress responses by the vector (Baxter et al., 2017; Rosche et al., 2021). In addition, viruses could trigger these responses as a way to manipulate the vector's ability to spread (Hurd, 2003; Targett, 2006). While the former mechanism is borne out by our data (see discussion of stress and antiviral pathways above), we can make several predictions about possible manipulation of vectors by the viruses. First, we would expect a more viral species-specific response by the vector, rather than a generalized response caused by increased virus titre. Second, we predict that the effect of the virus on the vector will evolve rapidly, with closely related viruses showing different effects on the vector. We explore these predictions below. 
Species-specificity of vector-virus interactions.

322 Viruses indeed show different patterns of interaction with varroa that seem linked to their

323 ecology. For example, some of the more pathogenic bee viruses (DWVa, DWVc and BQCV)

324 interact with the same module in the host gene expression network (Module 13, Fig 2b).

325 These viruses are known to be associated with varroa infestation (Benjeddou et al., 2001;

326 Daughenbaugh et al., 2015; Kevill et al., 2017; McMenamin \& Genersch, 2015), and have a

327 positive correlation with each other (Fig 2a). A different set of modules is affected by

328 viruses that show a high level of expression in varroa, though are detected only at low

329 levels in honey bees (VDV2, ARV-2, and VOV-1 (Fig 2a)) (Chen et al., 2021; S. Levin et al.,

330 2016a, 2017), suggesting that these viruses may be infecting the mites, rather than using

331 them as a vector. Interestingly, DWVa, the major driver of bee population declines, showed

332 strong interactions with the varroa gene expression network, but modules associated with

333 stress and antiviral responses were largely unaffected, suggesting that this virus may avoid

334 them (Fig 2b). This could be due to the fact that the virus does not replicate in varroa

335 (Gisder \& Genersch, 2020), though it does trigger a number of other gene expression

336 responses. These data suggest that viruses interact with varroa in diverse ways and that

337 varroa gene expression is not likely solely driven by their titre.

Vector-virus interactions modified by a minute change in viral sequence.

340 Virus interaction with its host can drive viral evolution and speciation (Koskella \&

341 Brockhurst, 2014), as host shift is a main force in viral diversification (Geoghegan et al.,

342 2017; Ricklefs et al., 2014), and can change the pathogen virulence (Williams \& Kamel,

343 2018). Our findings show that this also can occur in vector-virus interactions, as we found

344 that closely related viruses have distinctly different effects on the vector's gene expression.

345 The two most well known variants of the DWV swarm, DWVa and DWVb are associated 346 with varroa mites and colony collapses (Martin \& Brettell, 2019). Varroa infection drives

347 the DWV swarm evolution and the transition between the two variants (Dalmon et al.,

348 2017; Gisder et al., 2018). Despite high sequence similarity, DWVb is less associated with

349 the mite's infestation level in the colony, compared to DWVa (Norton et al., 2021), while on

350 the other hand DWVb is the only strain with concrete evidence for replicating in the mite

351 (Gisder \& Genersch, 2020). We therefore expected that the two variants, although highly 
similar in sequence, will have different interaction with the mite's gene regulatory networks. Indeed, we found that the two variants show contrasting interactions with the vector modules: while DWVa shows both positive and negative interactions with the mite's modules, DWVb shows no significant interactions with varroa modules (Fig 2b), which is surprising since DWVb was shown to replicate in the mite (Gisder \& Genersch, 2020) and should therefore interact with the vector genes. However, we should note that the analysis can be biased by viruses with high titers, such as DWVa, which extreme high viral loads in many samples may mask other virus-module and virus-virus interactions. Our findings suggest that a small modification in the virus's sequence can lead to a great change in the virus interaction with its vector, and that the vector-virus interactions are continuously and rapidly changing, resulting in a diverse viral community. Experimental infections of varroa by individual viruses can help further refine how individual viruses interact with the vector.

\section{Co-infecting viruses do not compete in the vector}

367 The 'competitive exclusion principle' states that when competing in the same niche, one 368 species will always suppress the other (Domingo, 2016). This was demonstrated also to happen in vectors, at least in mosquito cell-lines co-infected with two viruses (Abrao \& da Fonseca, 2016; Pepin et al., 2008). In the light of the vector being a limited nourishing

371 source, we could have expected the multi-infecting viruses to compete with each other in

372 the varroa mite. However, we found exactly the opposite, and the only significant intra-

373 viral interactions are positive ones (Fig 2a). This result, along with the high viral diversity

374 in the varroa mite, support the model by Leeks et al (Leeks et al., 2018), which suggests

375 that beneficial multi-viral interactions help to maintain high viral diversity. Still, our

376 findings do not exclude the 'short-sighted evolution' model of virulence, which argues that

377 in diverse infections, faster growing (more virulent) strains are favoured because they

378 compete for limited resources (B. R. Levin \& Bull, 1994). As the virulence of these viruses to

379 varroa mite were not tested so far, we cannot conclude at this point the evolutionary

380 mechanism which led to the observed multi-viral dynamic in the mite.

381 The positive correlation between some of the viruses may even imply mutualistic

382 interactions, a phenomenon observed before for different strains of West Nile virus co- 
383 infecting mosquito (Ciota et al., 2012). A few mechanisms for mutualistic virus-virus

384 relationships were suggested before such as cross-immunity, in multi-viral infection of

385 influenza and other respiratory viral diseases (Ferguson et al., 2003; Nickbakhsh et al.,

386 2019), and structural protein complementation in measles virus (Shirogane et al., 2012,

387 2016), and in mutants of Dengue virus infecting mosquito cells (Aaskov et al., 2006).

388 However, the latter is the only example for vector-borne viruses. As viral colonization is the

389 bottleneck for transmission, cooperative interaction between viruses in the vector have

390 direct implications for the viral community dynamics, as they can favour specific viral

391 strains that are not necessarily more virulent in a single, or even double infected vector.

392 This further emphasizes the need to study multi-viral infections and their molecular

393 mechanism.

395 Our study contributes to the ongoing investigation of the way viruses interact with their

396 vector, and how this affects the disease course, specifically multi-viral infection, a current

397 major gap in vector-borne viral research (Ciota, 2019; Vogels et al., 2019). Our results

398 imply a link between the virus epidemiological traits and its relationship with the vector. In

399 addition, not only that co-occurring viruses interact with each other, but their abundance

400 may predict the way these viruses will regulate their vector, and potentially, its ability to

401 successfully infect a new host. However, experiments on these viruses' biology and effect

402 on the vector are needed to have the full ecological context of these findings. Hopefully, the

403 gene network pipeline established here can be adopted to other vector-borne diseases,

404 opening the way to study the vector and its associated pathogenic and mutualistic

405 symbionts (Hegde et al., 2015; Rainey et al., 2014). 


\section{Materials and methods:}

407 In this study we investigated virus-virus and vector-virus interactions by two approaches:

408 (1) meta-transcriptomic and (2) gene-silencing experiments. In the meta-transcriptomic 409 analysis, we looked at the overall viral landscape in the different libraries, detected vector 410 modules (co-expressing genes using gene-network analysis), and correlated the viruses' 411 abundance to the vector modules. Last, we tested if the virus abundance matrix can predict 412 the virus-vector interaction. In the second step of the study, we experimentally validated 413 the vector-virus interaction on specific virus-gene combinations, selected based on the 414 meta-transcriptomic analysis, by RNAi-silencing varroa hub-genes and measuring the viral 415 load. All analyses were carried out in the R statistical environment (Team, 2013). All meta416 transcriptomic analyses are available and reproducible directly from the online

417 supplementary data: https://github.com/nurit-eliash/varroa-virus-networks.

(a) Vector-virus interaction, meta-transcriptomic analysis

420 Sequence Read Archive (SRA) data collection

421 To obtain varroa RNAseq data, we searched for "varroa" term in the SRA databases (NCBI, 422 January 2020) with the following filtering criteria: "RNA" (Source filter), "RNA-seq"

423 (Library Strategy filter) using Illumina technology, and the terms "TRANSCRIPTOMIC" and

424 "VIRAL RNA" (Library Source filter). In total, the filtration yielded 71 libraries of varroa

425 transcriptomes from 11 different studies. The libraries vary significantly in mite species,

426 library preparation method and total library size, number of mites per sample, collected 427 geographical region, sex, physiological stage, and the host species and developmental stage 428 from which the mite was collected (for libraries details please see table S15).

Reads mapping and transcripts quantification

431 The reads were mapped to both available varroa genome (Vdes_3.0, accession number:

432 GCF_002443255 (Techer et al., 2019)), and to the genomes of 23 selected viruses (Table 1).

433 The alignment and estimation of transcript and virus abundances in transcripts per million 434 (TPM), was carried out using Kallisto (Bray et al., 2016) (version 0.46.1 with default 
options). Kallisto pseudo-aligned reads to 35, 659 identified varroa isoforms from 10, 247 genes.

438 The viruses were selected following a survey in the literature and NCBI genome database

439 for viruses related to honey bee and/or varroa mite. Among the hundreds of virus

440 sequences, we included only viruses that were previously detected in varroa, in addition to

441 honey bee-pathogenic viruses (Table 1). The final 23 viruses are mostly positive ssRNA

442 viruses, three are negative ssRNA viruses (ARV-2 (Rhabdoviridae), VOV-1

443 (Orthomyxoviridae) and VDV4 (unclassified)), and another three are DNA viruses (one 444 circular dsDNA filamentous, and two ssDNA, found only in varroa mite and not in honey 445 bees (Kraberger et al., 2018)). Although recently many DNA viruses were found in bees

446 (Kraberger et al., 2019), their abundance and importance to varroa/bee health is unknown.

447 Therefore, these sequences were not included in the current study.

Filtering data set

450 Given the diversity of sources, we wanted to make sure that the input data were as

451 homogeneous as possible. A PCA of the different libraries based on varroa gene expression

452 showed that five libraries are obvious outliers (Fig S2a). These were excluded from further

453 analysis: library SRR8867385 (Brettell et al., 2019); libraries SRR5109825 and

454 SRR5109827 from (Remnant et al., 2017), were deep sequenced for small RNA; and library

455 SRR3927496 from a study by (Levin et al., 2016), in which a specific virome extraction

456 procedure was implemented prior to library preparation. Last, library SRR533974 by

457 (Cornman et al., 2013), was made of a pull of 1,000 mites, which is exceptionally higher

458 than the number of mites used in most of the studies (a pool of one - five mites). All of these

459 exceptional sample preparation procedures may account for these libraries' deviation from

460 the majority of the data sets in the PCA plot. The remaining 66 libraries are distributed

461 somewhat homogeneously in a subsequent PCA (Fig S2b), and their reads were used for

462 further analyses. 
Viral abundance analysis

465 Viral abundance for all 23 viruses revealed that although reported before in varroa, five viruses were not detected in any of the libraires (CBPV, AFV, ANV, VPVl_46 and VPVL_36)

467 (Fig 1), and so were not included in further analyses. To check for virus-virus interactions 468 within the mite, we conducted correlation matrix abundance using Pearson correlation 469 method for all viruses' pairs on the log10 transformed TPM (Fig 2a).

Weighted gene network co-expression analysis (WGCNA)

472 We used a network analysis approach to identify groups of genes that share a similar 473 expression pattern across a large set of available varroa transcriptomic data (RNAseq). To 474 construct the gene-network, a weighted gene co-expression analysis was carried out using 475 the WGCNA package in R, following the authors' tutorial (Langfelder \& Horvath, 2008, 476 2016). The WGCNA included 4 main steps: (1) Network construction and module detection;

477 (2) Correlating modules to external information, the varroa viral load; (3) Identifying

478 important genes; and (4) GO-term enrichment analysis for varroa modules which interact 479 with the viral load.

(1) Network construction and module detection

481 Based on the analysis of network topology, for the construction of the network we set our 482 threshold for merging of modules to 0.25 , minimum number of 30 genes per module, and 483 the power $\beta$ of 12 . This power is the lowest for which the scale-free topology fit index curve 484 flattens out upon reaching a high value, in this case, when Rsq reaches 0.886 (Fig S1a). We 485 then performed hierarchical clustering of the genes based on topological overlap (sharing 486 of network neighborhood) to identify groups of genes who coexpressed across libraries, 487 these are the network modules (Fig S1b).

489 To test if the varroa modules interact with the different viruses it carries, we correlated the 490 module eigengenes to the viruses' load (log10TPM). We used Pearson correlation method 491 and adjusted the p-values for multiple comparisons using the Benjamini-Hochberg method 492 to control the false discovery rate (Benjamini \& Hochberg, 1995) (Fig 2b). 
493 (3) Identifying hub genes in network modules

494 Hub genes have high connectivities within a module (Module Membership) (Langfelder \&

495 Horvath, 2008), and their annotation (based on sequence similarity to homologous genes).

496 The Module Membership is calculated by Pearson correlation of the module eigengene and

497 the gene expression. Genes with high Module Membership and relevant annotation are

498 likely to play a role in the vector-virus interaction and are good candidates for later

499 experimental validation.

(4) GO-term enrichment analysis for varroa modules

501 GO-terms enrichment analyses for the genes in the significantly interacting modules, were

502 conducted with R package GOstats using the hypergeometric test for association of

503 categories and genes (Falcon \& Gentleman, 2007). The test parameters for each species and

504 each ontology (biological process - BP) using gene ID from NCBI were as follow: p-value

505 cutoff $<0.05$, not conditional and with detection of over-represented G0 terms

506 (testDirection = over). All analyses are available and can be reproduced directly from the

507 online supplementary data on GitHub: https://github.com/nurit-eliash/varroa-virus-

508 networks.

Correlating virus interaction and virus-varroa interactions

511 To test if we can predict the virus-varroa interaction given the virus abundance, we used

512 Mantel-test for correlation between two distance-matrices (Mantel, 1967).

514 (b) Vector-virus interaction, mites' gene - silencing experiment

515 In the second step of the study, we experimentally validated specific gene-virus

516 interactions, as predicted by the meta-transcriptomic model. To check if indeed the gene

517 expression is correlated to the varroa viral load, we silenced hub genes with high Module

518 Membership and relevant annotation (see section '(3) Identifying hub genes in network

519 modules' in the meta-transcriptomic method part). We then checked for the viral load in

520 the silenced mites. For the list of the selected genes for silencing, their accession and

521 annotation please see Table S12. 


\section{Mites and honey bee collection}

523 Mites and bees (A. mellifera liguistica) were collected from the same colonies, at the apiary 524 of Okinawa institute of science and technology (OIST). The hives were not treated against

525 mites and were supplemented with sugar solution and 70\% pollen cakes as necessary.

526 Mites were collected from drone and worker pupa of different stages and were kept on

527 bees until soaking, up to five hours from collection.

RNAi silencing of varroa genes

DsRNA preparation

531 For the dsRNA preparation, we first synthesized a T7-promotor attached dsDNA of each of

532 the targeted genes by PCR amplification of cDNA prepared from a pool of 5-10 mites, with

533 specific primers with T7-promotor attached to the 5' end (see Table S16 for the primers

534 information, and section 'varroa genes primer design' for details on primer design). For

535 PCR amplification we used Phusion ${ }^{\mathrm{TM}}$ High-Fidelity DNA Polymerase (Thermo Fisher

536 Scientific) in a PTC-200 Peltier Thermal Cycler, MJ Research (BioRad, Toronto, Ontario)

537 with the following steps: an initial denaturation at $98{ }^{\circ} \mathrm{C}$ for 30 secs followed by 30 cycles

538 of denaturation at $98^{\circ} \mathrm{C}$ for $10 \mathrm{sec}$, annealing at $60{ }^{\circ} \mathrm{C}$ for $10 \mathrm{sec}$, an extension at $72{ }^{\circ} \mathrm{C}$ for

$53930 \mathrm{sec}$, and a final extension of $72{ }^{\circ} \mathrm{C}$ for additional 5 mins. We checked that the size of the

540 amplicons matches the expected length, by running $3 \mu \mathrm{l}$ of the PCR product in 1\% agarose

541 gel $(135 \mathrm{~V}, 20 \mathrm{~min})$, then verified the sequence by purifying the product and Sanger

542 sequencing on ThermoFisher SeqStudio Genetic Analyzer, using the original reverse primer

543 and BigDye® Direct Cycle Sequencing kit (Thermo Fisher Scientific), following

544 manufacturer's instructions. Prior to dsRNA synthesis, we purified the PCR products using

545 MinElute PCR purification kit (QIAGEN), measured their concentration by Qubit ${ }^{\mathrm{TM}} 4$

546 Fluorometer (Life Technologies) with dsDNA HS Assay Kit (Invitrogen), and checked the

547 product size by 4200 Tapestation (Agilent, Tokyo, Japan).

548 Next, 1200ng of the purified dsDNA with T7-promotor attached was used as a template for

549 the dsRNA synthesis, using MEGAscript ${ }^{\text {TM }}$ T7 Transcription Kit (Thermo Fisher Scientific).

550 We followed the manufacturer's protocol, with slight adjustments. The mix in total volume

551 of $100 \mathrm{ul}$ was incubated overnight at $37^{\circ} \mathrm{C}$, following the addition of TURBO DNase buffer to 
552 the reaction, and incubation for another 15 mins at $37^{\circ} \mathrm{C}$. We purified and concentrated the

553 RNA mix using MEGAclear Transcription Clean-Up kit (Thermo Fisher Scientific), and

554 finally measured the RNA concentration by Nanodrop, and checked the product size by

5554200 Tapestation (Agilent, Tokyo, Japan). To make sure that the dsRNA effect is specific, we

556 also prepared a negative control dsRNA of a non-target gene, green fluorescent protein

557 (GFP), using pET6Xhn-GFPuv vector (Clontech, Takara) as a template.

559 For applying the dsRNA into the mite body, we followed a protocol first developed by

560 (Campbell et al., 2010), and successfully done by us and others (Garbian et al., 2012; B. T.

561 Nganso et al., 2021; Singh et al., 2016). We added three mites in a $0.5 \mathrm{ml}$ tube containing

$56220 \mu \mathrm{l}$ dsRNA $(2.5 \mu \mathrm{g} / \mu \mathrm{l})$ in $0.9 \% \mathrm{NaCl}$ solution. The tubes were kept in $4{ }^{\circ} \mathrm{C}$ for $10-15$ mins

563 then we checked that all of the mites were soaked, and re-dipped them into the solution, if

564 needed. The mites were kept in $4{ }^{\circ} \mathrm{C}$ overnight ( $\sim 16$ hours), dried on a filter paper, then put

565 on a bee pupa (all same age), three mites per bee in a gelatin capsule with perforated top

566 (\#1, 0.49ml, HF capsules, Matuya, Japan). Following former studies (Campbell, Budge, et al.,

567 2016; Singh et al., 2016), showing optimal silencing effectiveness 48 hours post dsRNA

568 treatment, the mites were incubated for 48 hours in a controlled, dark environment at

$56934.5^{\circ} \mathrm{C}, 60-75 \% \mathrm{RH}$, and the pupa was replaced after 24 hours. After incubation, each

570 moving-viable mite was separated in a 1.5-ml tube, snap-frozen in liquid nitrogen and kept

571 in $-80^{\circ} \mathrm{C}$ until RNA extraction. The experiment was replicated in seven experimental

572 batches, between October - November 2020, and each batch included control group mites

573 soaked in GFP-dsRNA of the same concentration and kept under the same conditions as

574 described above. We checked the effect of the dsRNA treatment on mite viability, by

575 comparing the numbers of live and dead dsRNA-treated mites to that of the control mites in

576 each experimental batch (Fisher exact test for goodness of fit, p < 0.05) (table S14).

578 RNA extraction and cDNA preparation

579 Each individual mite was processed following the protocol developed in our lab and

580 described previously (Hasegawa et al., 2021). Briefly, each individual mite was crushed in a

$581 \quad 1.5 \mathrm{ml}$ tube dipped in liquid nitrogen, then RNA was extracted using slightly modified 
582 TRIzol manufacturer's protocol, with 50\% volume of reagents. Total RNA quality and

583 quantity were evaluated using Nanodrop spectrophotometer. 300ng of purified RNA was

584 used to synthesize a first-strand cDNA using SuperScript II (Invitrogen) and 1:2 ratio of

585 random hexamer and oligo dT primers following the manufacturer's protocol.

Measuring varroa gene expression and viral load

588 For both viruses and genes, sets of primers were designed with NCBI primer design tool

589 (utilizing Primer3 and BLAST), with default parameters and product size set to 100 -

590 400bp. Primer's sequence, product length size and gene IDs or viruses' accession numbers

591 are provided in Tables S16 and S17 for varroa genes and viruses respectively. The product

592 size of all amplicons was checked by running in 1\% agarose gel (135V, $20 \mathrm{~min})$.

593 Varroa genes primer design

594 For each of the varroa selected genes (Table S12) we designed two sets of primers, using

595 the gene mRNA sequence as a template. A first set for dsRNA preparation (as described in

596 'DsRNA preparation' section), and a second, not overlapping primers-set for gene

597 quantification using qPCR.

598 Identification of local viruses and RdRp primer design

599 We targeted the conserved gene of RNA-dependent RNA polymerase (RdRp) commonly

600 used for detection and measurement of different RNA-viruses in honey bees and varroa

601 mites (de Miranda et al., 2013; Gisder et al., 2018). As the genome sequence of bee viruses

602 is slightly different across geographical regions (Cornman et al., 2013; Gisder et al., 2018;

603 Gisder \& Genersch, 2021), we first wanted to obtain the specific sequence of the strain

604 present in our local mites. For that, for each of the three viruses (VDV2, ARV-2 and DWVa),

605 we amplified and sequenced a wide region of the RdRp gene (amplicon size 800bp) (see

606 section 'DsRNA preparation' for PCR and sequencing details). To verify the sequence, we

607 nBlasted the product to the nr database (NCBI) (Altschul et al., 1990). The reverse

608 complementary sequence of viral RdRp was then used as a template to design the qPCR

609 primer sets, as described above. For viruses' amplicon sequences, nBlast results and primer

610 sequence and position, see Data S1. 
611 qPCR

612 To evaluate the relative gene expression and viral load we performed a qPCR using a

613 StepOnePlus Real-Time PCR system (Applied Biosystems Japan, Tokyo, Japan) with TB

614 Green® Premix Ex Taq ${ }^{\text {TM }}$ II (Tli RNaseH Plus, Takara). The cycling conditions were as

615 follows: $95^{\circ} \mathrm{C}$ for 30 secs, followed by 40 cycles at $95^{\circ} \mathrm{C}$ for 5 seconds, and $60{ }^{\circ} \mathrm{C}$ for 30

616 secs. Data normalization and quantitating was done using StepOnePlus Real-Time system

617 software (Applied Biosystems, Japan), with automatic threshold set. Small subunit of the

618 ribosomal RNA gene (18S rRNA) of varroa was used as a normalizing gene (Campbell et al.,

619 2016), and a control mite (treated with GFP-dsRNA) from the same experimental batch,

620 used as the normalizing sample. For all qPCR assays a no-template control was included

621 (data not shown). To test for differences in gene expression and viral load of dsRNA treated

622 mites and control mites, we used a-parametric Wilcoxon signed-ranks test. For all

623 statistical tests, the p-values were adjusted for multiple comparisons using the Benjamini-

624 Hochberg method to control the false discovery rate (Benjamini \& Hochberg, 1995).

625

626 Acknowledgments

627 We wish to thank Dr. Olesya Gusachenko (University of St Andrews, UK) for consulting

628 regarding the virus identification and viruses' primer design. We also wish to thank Dr.

629 Maeva Techer for her help in the tedious task of mites' collection for the silencing

630 experiment, and for all Mikheyev unit members at OIST for stimulating discussion and

631 support throughout.

632

633 Competing interests

634 The authors declare that they have no competing interests. 


\section{References}

636 Aaskov, J., Buzacott, K., Thu, H. M., Lowry, K., \& Holmes, E. C. (2006). Long-term transmission of 637 defective RNA viruses in humans and Aedes mosquitoes. Science, 311(5758), 236-238.

638 Abrao, E. P., \& da Fonseca, B. A. L. (2016). Infection of Mosquito Cells (C6/36) by Dengue-2 Virus 639 Interferes with Subsequent Infection by Yellow Fever Virus. Vector Borne and Zoonotic $640 \quad$ Diseases , 16(2), 124-130.

641 Alcaide, C., Rabadán, M. P., Moreno-Pérez, M. G., \& Gómez, P. (2020). Implications of mixed viral infections on plant disease ecology and evolution. Advances in Virus Research, 106, 145-169.

643 Altschul, S. F., Gish, W., Miller, W. T., Myers, E. W., \& Lipman, D. J. (1990). Basic local alignment search tool. Journal of Molecular Biology, 215(3), 403-410.

Batovska, J., Mee, P. T., Lynch, S. E., Sawbridge, T. I., \& Rodoni, B. C. (2019). Sensitivity and specificity of metatranscriptomics as an arbovirus surveillance tool. Scientific Reports, 9(1), 19398.

647 Batson, J., Dudas, G., Haas-Stapleton, E., Kistler, A. L., Li, L. M., Logan, P., Ratnasiri, K., \& Retallack, H.

648 (2021). Single mosquito metatranscriptomics identifies vectors, emerging pathogens and reservoirs in one assay. eLife, 10. https://doi.org/10.7554/eLife.68353

Baxter, R. H. G., Contet, A., \& Krueger, K. (2017). Arthropod Innate Immune Systems and VectorBorne Diseases. Biochemistry, 56(7), 907-918.

Benjamini, Y., \& Hochberg, Y. (1995). Controlling the False Discovery Rate: A Practical and Powerful Approach to Multiple Testing. Journal of the Royal Statistical Society. Series B, Statistical

Benjeddou, M., Leat, N., Allsopp, M., \& Davison, S. (2001). Detection of acute bee paralysis virus and black queen cell virus from honeybees by reverse transcriptase pcr. Applied and Environmental Microbiology, 67(5), 2384-2387.

Blair, C. D., \& Olson, K. E. (2015). The role of RNA interference (RNAi) in arbovirus-vector interactions. Viruses, 7(2), 820-843. 
660

661

662

663

664

665

666

667

668

Brault, A. C., Savage, H. M., Duggal, N. K., Eisen, R. J., \& Staples, J. E. (2018). Heartland virus epidemiology, vector association, and disease potential. In Viruses (Vol. 10, Issue 9, p. 498). MDPI AG. https://doi.org/10.3390/v10090498

Bray, N. L., Pimentel, H., Melsted, P., \& Pachter, L. (2016). Near-optimal probabilistic RNA-seq quantification. Nature Biotechnology, 34(5), 525-527.

Brettell, L. E., Schroeder, D. C., \& Martin, S. J. (2019). RNAseq analysis reveals virus diversity within hawaiian apiary insect communities. Viruses, 11(5). https://doi.org/10.3390/v11050397

Campbell, E. M., Budge, G. E., \& Bowman, A. S. (2010). Gene-knockdown in the honey bee mite Varroa destructor by a non-invasive approach: studies on a glutathione S-transferase. Parasites \& Vectors, 3,73 .

Campbell, E. M., Budge, G. E., Watkins, M., \& Bowman, A. S. (2016). Transcriptome analysis of the synganglion from the honey bee mite, Varroa destructor and RNAi knockdown of neural peptide targets. Insect Biochemistry and Molecular Biology, 70, 116-126.

Campbell, E. M., McIntosh, C. H., \& Bowman, A. S. (2016). A Toolbox for Quantitative Gene Expression in Varroa destructor: RNA Degradation in Field Samples and Systematic Analysis of Reference Gene Stability. PloS One, 11(5), e0155640.

Carreck, N. L., Ball, B. V., \& Martin, S. J. (2010). Honey bee colony collapse and changes in viral prevalence associated with Varroa destructor. Journal of Apicultural Research, 49(1), 93-94.

Chen, G., Wang, S., Jia, S., Feng, Y., Hu, F., Chen, Y., \& Zheng, H. (2021). A New Strain of Virus Discovered in China Specific to the Parasitic Mite Varroa destructor Poses a Potential Threat to Honey Bees. Viruses, 13(4), 679.

Cime-Castillo, J., Delannoy, P., Mendoza-Hernández, G., Monroy-Martínez, V., Harduin-Lepers, A., Lanz-Mendoza, H., Hernández-Hernández, F. de la C., Zenteno, E., Cabello-Gutiérrez, C., \& RuizOrdaz, B. H. (2015). Sialic acid expression in the mosquito Aedes aegypti and its possible role in dengue virus-vector interactions. BioMed Research International, 2015, 504187. 
Ciota, A. T. (2019). The role of co-infection and swarm dynamics in arbovirus transmission. Virus Research, 265, 88-93.

Ciota, A. T., Ehrbar, D. J., Van Slyke, G. A., Willsey, G. G., \& Kramer, L. D. (2012). Cooperative interactions in the West Nile virus mutant swarm. BMC Evolutionary Biology, 12, 58.

Cornman, R. S., Boncristiani, H., Dainat, B., Chen, Y., VanEngelsdorp, D., Weaver, D., \& Evans, J. D. (2013). Population-genomic variation within RNA viruses of the Western honey bee, Apis mellifera, inferred from deep sequencing. BMC Genomics, 14(1), 154.

Dalmon, A., Desbiez, C., Coulon, M., Thomasson, M., Le Conte, Y., Alaux, C., Vallon, J., \& Moury, B. (2017). Evidence for positive selection and recombination hotspots in Deformed wing virus (DWV). Scientific Reports, 7. https://doi.org/10.1038/srep41045

Daughenbaugh, K. F., Martin, M., Brutscher, L. M., Cavigli, I., Garcia, E., Lavin, M., \& Flenniken, M. L. (2015). Honey bee infecting Lake Sinai viruses. Viruses, 7(6), 3285-3309. Torina, A., Ayllón, N., Vancova, M., Golovchenko, M., Grubhoffer, L., ... Rego, R. O. M. (2017). Tick-Pathogen Interactions and Vector Competence: Identification of Molecular Drivers for Tick-Borne Diseases. Frontiers in Cellular and Infection Microbiology, 7, 114.

de Miranda, J. R., Bailey, L., Ball, B. V., Blanchard, P., Budge, G. E., Chejanovsky, N., Chen, Y.-P., Research, 52(4), 1-56.

Deshoux, M., Monsion, B., \& Uzest, M. (2018). Insect cuticular proteins and their role in transmission of phytoviruses. Current Opinion in Virology, 33, 137-143.

Díaz-Muñoz, S. L. (2017). Viral coinfection is shaped by host ecology and virus-virus interactions across diverse microbial taxa and environments. Virus Evolution, 3(1), vex011. 
710

711

712

713

714

715

716

717

718

719

720

721

722

723

724

725

726

727

728

729

730

731

732

733

734

Domingo, E. (2016). Chapter 6 - Virus Population Dynamics Examined with Experimental Model

Systems. In E. Domingo (Ed.), Virus as Populations (pp. 197-225). Academic Press.

Erez, Z., Steinberger-Levy, I., Shamir, M., Doron, S., Stokar-Avihail, A., Peleg, Y., Melamed, S., Leavitt, A., Savidor, A., Albeck, S., Amitai, G., \& Sorek, R. (2017). Communication between viruses guides lysis-lysogeny decisions. Nature, 541(7638), 488-493.

Falcon, S., \& Gentleman, R. (2007). Using GOstats to test gene lists for GO term association. Bioinformatics , 23(2), 257-258.

Ferguson, N. M., Galvani, A. P., \& Bush, R. M. (2003). Ecological and immunological determinants of influenza evolution. Nature, 422(6930), 428-433.

Garbian, Y., Maori, E., Kalev, H., Shafir, S., \& Sela, I. (2012). Bidirectional transfer of RNAi between honey bee and Varroa destructor: Varroa gene silencing reduces Varroa population. PLoS Pathogens, 8(12), e1003035.

Geoghegan, J. L., Duchêne, S., \& Holmes, E. C. (2017). Comparative analysis estimates the relative frequencies of co-divergence and cross-species transmission within viral families. PLoS Pathogens, 13(2), e1006215.

Gisder, S., \& Genersch, E. (2020). Direct evidence for infection of Varroa destructor mites with the bee-pathogenic deformed wing virus variant B - but not variant A - via fluorescence-in situhybridization analysis. Journal of Virology.

Gisder, S., \& Genersch, E. (2021). Direct Evidence for Infection of Varroa destructor Mites with the Bee-Pathogenic Deformed Wing Virus Variant B - but Not Variant A - via Fluorescence-in situHybridization Analysis. Journal of Virology. https://doi.org/10.1128/JVI.01786-20

Gisder, S., Möckel, N., Eisenhardt, D., \& Genersch, E. (2018). In vivo evolution of viral virulence: switching of deformed wing virus between hosts results in virulence changes and sequence shifts. Environmental Microbiology, 20(12), 4612-4628.

Goenaga, S., Kenney, J. L., Duggal, N. K., Delorey, M., Ebel, G. D., Zhang, B., Levis, S. C., Enria, D. A., \& 
Brault, A. C. (2015). Potential for Co-Infection of a Mosquito-Specific Flavivirus, Nhumirim Virus, to Block West Nile Virus Transmission in Mosquitoes. Viruses, 7(11), 5801-5812.

Göertz, G. P., van Bree, J. W. M., Hiralal, A., Fernhout, B. M., Steffens, C., Boeren, S., Visser, T. M., the United States of America, 116(38), 19136-19144.

Göertz, G. P., Vogels, C. B. F., Geertsema, C., Koenraadt, C. J. M., \& Pijlman, G. P. (2017). Mosquito coinfection with Zika and chikungunya virus allows simultaneous transmission without affecting vector competence of Aedes aegypti. PLoS Neglected Tropical Diseases, 11(6), e0005654.

Hasegawa, N., Techer, M., \& Mikheyev, A. S. (2021). A toolkit for studying Varroa genomics and

Hegde, S., Rasgon, J. L., \& Hughes, G. L. (2015). The microbiome modulates arbovirus transmission transcriptomics: preservation, extraction, and sequencing library preparation. BMC Genomics, in mosquitoes. Current Opinion in Virology, 15, 97-102.

Huang, Y.-J. S., Higgs, S., \& Vanlandingham, D. L. (2019). Arbovirus-Mosquito Vector-Host

Hurd, H. (2003). Manipulation of medically important insect vectors by their parasites. Annual Review of Entomology, 48, 141-161. behavior in Aedes triseriatus and Aedes albopictus (Diptera: Culicidae). Journal of Medical Entomology, 49(6), 1424-1429. 
Overwinter Colony Losses of European Honey Bees. Viruses, 9(11). https://doi.org/10.3390/v9110314

Koskella, B., \& Brockhurst, M. A. (2014). Bacteria-phage coevolution as a driver of ecological and evolutionary processes in microbial communities. FEMS Microbiology Reviews, 38(5), 916-931. Infectious Diseases, 71, 179-188.

Kraberger, S., Visnovsky, G. A., van Toor, R. F., Male, M. F., Waits, K., Fontenele, R. S., \& Varsani, A. (2018). Genome Sequences of Two Single-Stranded DNA Viruses Identified in Varroa destructor. Genome Announcements, 6(9). https://doi.org/10.1128/genomeA.00107-18

Langfelder, P., \& Horvath, S. (2008). WGCNA: An R package for weighted correlation network analysis. BMC Bioinformatics, 9. https://doi.org/10.1186/1471-2105-9-559

773 Langfelder, P., \& Horvath, S. (2016). Tutorials for WGCNA R package.

Leeks, A., Segredo-Otero, E. A., Sanjuán, R., \& West, S. A. (2018). Beneficial coinfection can promote

Levin, B. R., \& Bull, J. J. (1994). Short-sighted evolution and the virulence of pathogenic microorganisms. Trends in Microbiology, 2(3), 76-81. rhabdovirus- 1 in populations of pollinators and their parasites from two continents. Frontiers in Microbiology, 8(DEC), 2482. 
Levin, S., Sela, N., \& Chejanovsky, N. (2016b). Two novel viruses associated with the Apis mellifera pathogenic mite Varroa destructor. Scientific Reports, 6, 37710.

Luplertlop, N., Surasombatpattana, P., Patramool, S., Dumas, E., Wasinpiyamongkol, L., Saune, L.,

Maciel-de-Freitas, R., Koella, J. C., \& Lourenço-de-Oliveira, R. (2011). Lower survival rate, longevity and fecundity of Aedes aegypti (Diptera: Culicidae) females orally challenged with dengue virus serotype 2. Transactions of the Royal Society of Tropical Medicine and Hygiene, 105(8),

Mantel, N. (1967). The detection of disease clustering and a generalized regression approach. Cancer Research, 27(2), 209-220.

Martin, S. J., \& Brettell, L. E. (2019). Deformed wing virus in honeybees and other insects. Annual Review of Virology, 6(1).

Martin, S. J., Highfield, A. C., Brettell, L., Villalobos, E. M., Budge, G. E., Powell, M., Nikaido, S., \& Schroeder, D. C. (2012). Global honey bee viral landscape altered by a parasitic mite. Science,

McMenamin, A. J., \& Genersch, E. (2015). Honey bee colony losses and associated viruses. Current 336(6086), 1304-1306.

Moncayo, A. C., Edman, J. D., \& Turell, M. J. (2000). Effect of eastern equine encephalomyelitis virus on the survival of Aedes albopictus, Anopheles quadrimaculatus, and Coquillettidia perturbans (Diptera: Culicidae). Journal of Medical Entomology, 37(5), 701-706. induces Ixodes scapularis ticks to express an antifreeze glycoprotein gene that enhances their survival in the cold. The Journal of Clinical Investigation, 120(9), 3179-3190. 
810

811

812

813

814

815

816

817

818

819

820

821

822

823

824

825

826

827

828

829

830

831

832

833

834

Nganso, B. T., Mani, K., Eliash, N., Rafaeli, A., \& Soroker, V. (2021). Towards disrupting Varroa honey bee chemosensing: A focus on a Niemann-Pick type C2 transcript. Insect Molecular Biology. https://doi.org/10.1111/imb.12722

Nganso, B. T., Sela, N., \& Soroker, V. (2020). A genome-wide screening for RNAi pathway proteins in Acari. BMC Genomics, 21, 791.

Ng, J. C. K., \& Falk, B. W. (2006). Virus-vector interactions mediating nonpersistent and semipersistent transmission of plant viruses. Annual Review of Phytopathology, 44, 183-212.

Nickbakhsh, S., Mair, C., Matthews, L., Reeve, R., Johnson, P. C. D., Thorburn, F., von Wissmann, B., Reynolds, A., McMenamin, J., Gunson, R. N., \& Murcia, P. R. (2019). Virus-virus interactions impact the population dynamics of influenza and the common cold. Proceedings of the National Academy of Sciences of the United States of America. https://doi.org/10.1073/pnas.1911083116

Norton, A. M., Remnant, E. J., Tom, J., Buchmann, G., Blacquiere, T., \& Beekman, M. (2021). Adaptation to vector-based transmission in a honey bee virus. The Journal of Animal Ecology. https://doi.org/10.1111/1365-2656.13493

Olival, K. J., Hosseini, P. R., Zambrana-Torrelio, C., Ross, N., Bogich, T. L., \& Daszak, P. (2017). Host and viral traits predict zoonotic spillover from mammals. Nature, 546(7660), 646-650.

Pepin, K. M., Lambeth, K., \& Hanley, K. A. (2008). Asymmetric competitive suppression between strains of dengue virus. BMC Microbiology, 8, 28.

Rainey, S. M., Shah, P., Kohl, A., \& Dietrich, I. (2014). Understanding the Wolbachia-mediated inhibition of arboviruses in mosquitoes: progress and challenges. The Journal of General Virology, 95(Pt 3), 517-530.

Remnant, E. J., Shi, M., Buchmann, G., Blacquière, T., Holmes, E. C., Beekman, M., \& Ashe, A. (2017). A Diverse Range of Novel RNA Viruses in Geographically Distinct Honey Bee Populations. Journal of Virology, 91(16). https://doi.org/10.1128/JVI.00158-17 
835

836

837

838

839

840

841

842

843

844

845

846

847

848

849

850

851

852

853

854

855

856

857

858

859

Ricklefs, R. E., Outlaw, D. C., Svensson-Coelho, M., Medeiros, M. C. I., Ellis, V. A., \& Latta, S. (2014).

Species formation by host shifting in avian malaria parasites. Proceedings of the National Academy of Sciences of the United States of America, 111(41), 14816-14821.

Rocklöv, J., \& Dubrow, R. (2020). Climate change: an enduring challenge for vector-borne disease prevention and control. Nature Immunology, 21(5), 479-483.

Rosche, K. L., Sidak-Loftis, L. C., Hurtado, J., Fisk, E. A., \& Shaw, D. K. (2021). Arthropods Under Pressure: Stress Responses and Immunity at the Pathogen-Vector Interface. Frontiers in Immunology, 11, 629777.

Sanjuán, R., Illingworth, C. J. R., Geoghegan, J. L., Iranzo, J., Zwart, M. P., Ciota, A. T., Moratorio, G., Gago-Zachert, S., Duffy, S., \& Vijaykrishna, D. (2021). Five challenges in the field of viral diversity and evolution. https://aspace.repository.cam.ac.uk/bitstream/handle/1810/322603/fviro-01684949.pdf?sequence=2\&isAllowed=y

Schnettler, E., Tykalová, H., Watson, M., Sharma, M., Sterken, M. G., Obbard, D. J., Lewis, S. H., McFarlane, M., Bell-Sakyi, L., Barry, G., Weisheit, S., Best, S. M., Kuhn, R. J., Pijlman, G. P., ChaseTopping, M. E., Gould, E. A., Grubhoffer, L., Fazakerley, J. K., \& Kohl, A. (2014). Induction and suppression of tick cell antiviral RNAi responses by tick-borne flaviviruses. Nucleic Acids Research, 42(14), 9436-9446.

Shirogane, Y., Watanabe, S., \& Yanagi, Y. (2012). Cooperation between different RNA virus genomes produces a new phenotype. Nature Communications, 3, 1235.

Shirogane, Y., Watanabe, S., \& Yanagi, Y. (2016). Cooperative Interaction Within RNA Virus Mutant Spectra. In E. Domingo \& P. Schuster (Eds.), Quasispecies: From Theory to Experimental Systems (pp. 219-229). Springer International Publishing.

Singh, N. K., Eliash, N., Stein, I., Kamer, Y., Ilia, Z., Rafaeli, A., Soroker, V., Zeidman, I., Rafaeli, A., \& Soroker, V. (2016). Identification and gene-silencing of a putative odorant receptor transcription factor in Varroa destructor: possible role in olfaction. Insect Molecular Biology, 
25(2), 181-190.

861 Steinhauer, N., Kulhanek, K., Antúnez, K., Human, H., Chantawannakul, P., Chauzat, M. P., \& vanEngelsdorp, D. (2018). Drivers of colony losses. Current Opinion in Insect Science, 26, 142148.

Sutherst, R. W. (2004). Global change and human vulnerability to vector-borne diseases. Clinical Microbiology Reviews, 17(1), 136-173.

Targett, G. A. T. (2006). Parasites, arthropod vectors, and immune responses. Parasite Immunology, $28(4), 117-119$.

Team, R. C. (2013). R: A language and environment for statistical computing. https://www.Rproject.org/

Techer, M. A., Rane, R. V., Grau, M. L., Roberts, J. M. K., Sullivan, S. T., Liachko, I., Childers, A. K., Evans, J. D., \& Mikheyev, A. S. (2019). Divergent evolutionary trajectories following speciation in two ectoparasitic honey bee mites. Communications Biology, 2(1), 357. Arbovirus coinfection and co-transmission: A neglected public health concern? PLoS Biology, 17(1), e3000130.

Williams, P. D., \& Kamel, S. J. (2018). The evolution of pathogen virulence: Effects of transitions

Weaver, S. C., Charlier, C., Vasilakis, N., \& Lecuit, M. (2018). Zika, Chikungunya, and Other Emerging between host types. Journal of Theoretical Biology, 438, 1-8. J., McCallum, H., \& Fenton, A. (2017). What is a vector? Philosophical Transactions of the Royal Society of London. Series B, Biological Sciences, 372(1719). 
Wilson, A. L., Courtenay, O., Kelly-Hope, L. A., Scott, T. W., Takken, W., Torr, S. J., \& Lindsay, S. W. (2020). The importance of vector control for the control and elimination of vector-borne diseases. PLoS Neglected Tropical Diseases, 14(1), e0007831.

World Health Organization. (2017). Global vector control response: an integrated approach for the

$$
\text { en.pdf?ua=1 }
$$

891 Yañez, O., Piot, N., Dalmon, A., de Miranda, J. R., Chantawannakul, P., Panziera, D., Amiri, E., Smagghe,

$$
\text { Frontiers in Microbiology, 11, } 943 .
$$

894 Zink, S. D., Van Slyke, G. A., Palumbo, M. J., Kramer, L. D., \& Ciota, A. T. (2015). Exposure to West Nile 895 Virus Increases Bacterial Diversity and Immune Gene Expression in Culex pipiens. Viruses, $7(10), 5619-5631$.

Campbell, E. M., McIntosh, C. H., \& Bowman, A. S. (2016). A Toolbox for Quantitative Gene Expression in Varroa destructor: RNA Degradation in Field Samples and Systematic Analysis of Reference Gene Stability. PloS One, 11(5), e0155640. 